\title{
Nanotechnology and Solar Energy
}

\author{
M. S. A. Abdel-Mottaleb, ${ }^{1}$ J. A. Byrne, ${ }^{2}$ and D. Chakarov ${ }^{3}$ \\ ${ }^{1}$ NanoPhotochemistry and Solar Chemistry Lab, Department of Chemistry, Faculty of Science, Ain Shams University, \\ Cairo 11566, Egypt \\ ${ }^{2}$ Nanotechnology and Integrated BioEngineering Centre, School of Engineering, Faculty of Computing and Engineering, \\ University of Ulster, Newtownabbey BT37 OQB, UK \\ ${ }^{3}$ Department of Applied Physics, Chalmers University of Technology, 41296 Gothenburg, Sweden
}

Correspondence should be addressed to M. S. A. Abdel-Mottaleb, phochem08@photoenergy.org

Received 24 July 2011; Accepted 24 July 2011

Copyright () 2011 M. S. A. Abdel-Mottaleb et al. This is an open access article distributed under the Creative Commons Attribution License, which permits unrestricted use, distribution, and reproduction in any medium, provided the original work is properly cited.

Interest in solar energy conversion and the associated materials research and development has been inspired due to concerns regarding carbon dioxide emissions, job creation, and market instabilities due to the geopolitics and widespread consumption of fossil fuels. The wide introduction of solar powered devices made from nanomaterials has the potential to revolutionize global economic development. Increasing the efficiency and decreasing the costs of solar power are two key areas where nanoscience and nanotechnology can contribute most.

Nanotechnology has assumed a special status in semiconductor materials and catalysts. These two classes of materials could be considered as the key for solar energy conversion. Nanomaterials applied in solar energy conversion may be classified depending on their proposed application. Photocatalysts are a big family of nanomaterials (semiconductors or transition metal oxides) mostly applied in water treatment and solar water splitting for hydrogen production, among other useful applications.

Nanomaterials possess certain desirable properties like high catalytic activity, better stability in aqueous media, comparatively easier preparation techniques, and material economy. Still, nanomaterials suffer from certain drawbacks when they are used in photocatalytic and photoelectrochemical devices.

The fascinating optical properties of nanostructured materials find important applications in a number of solar energy utilization schemes and devices. Nanotechnology provides methods for fabrication and use of structures and systems with size corresponding to the wavelength of visible light. This opens a wealth of possibilities to explore the new, often of resonance character, phenomena observed when the object size and the electromagnetic field periodicity (light wavelength $\lambda$ ) match.

Titanium dioxide $\left(\mathrm{TiO}_{2}\right)$ is an n-type semiconductor that has attracted tremendous attention from researchers worldwide due to its potential applications in environmental protection and energy generation. It has demonstrated unique properties such as high adsorption ability and good photocatalytic activity. Dye-sensitized solar cells (DSSCs) are molecular system that consists of $\mathrm{TiO}_{2}$, an anchored molecular photosensitizer, a redox electrolyte, and a platinized photocathode. The sensitizers play a vital role in DSSCs, and a large number of Ru-complex sensitizers and organic sensitizers have been developed and tested. So far, sensitizers such as black dye, N719, and N3 are known as best sensitizers in DSSC. Black dye-sensitized nanocrystalline $\mathrm{TiO}_{2}$ solar cells have been reported to yield a solar to electric power conversion efficiency of over $11 \%$ under standard AM 1.5 conditions. Much effort has been made to increase photovoltaic performance (stability) of the DSSCs with the development of new sensitizers, electrodes, and nanostructured photoanode materials.

This special issue shows the current trends of research in this branch by presenting two reviews and 14 research articles covering several important areas, ranging from fundamental to applied topics.

\section{Acknowledgments}

The present issue would not be possible without the contribution of many persons. We would like to thank 
International Journal of Photoenergy and the staff, who have contributed to achieve the best possible quality for this publication. All authors of this issue, and many others, have enthusiastically responded to our call for papers and offered their best scientific knowledge. Around seventy top scientists and engineers in the field have been involved in the peer review of the papers. We wish to thank everyone for his or her contributions, which have made this special issue possible.

\section{S. A. Abdel-Mottaleb}

J. A. Byrne

D. Chakarov 


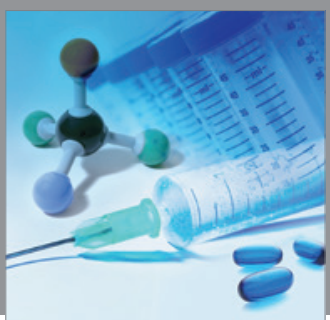

International Journal of

Medicinal Chemistry

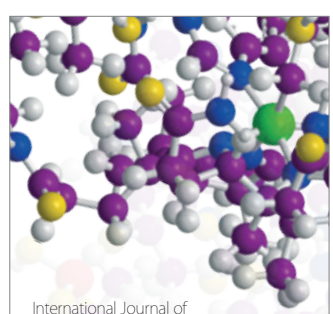

Carbohydrate Chemistry

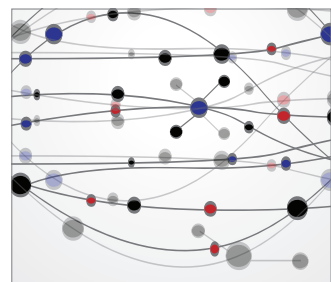

The Scientific World Journal
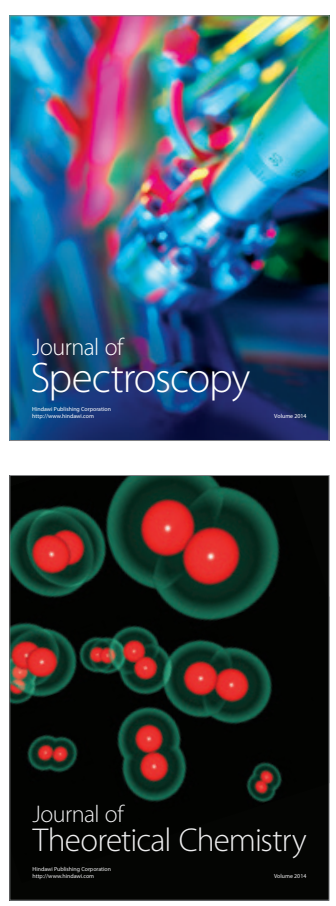
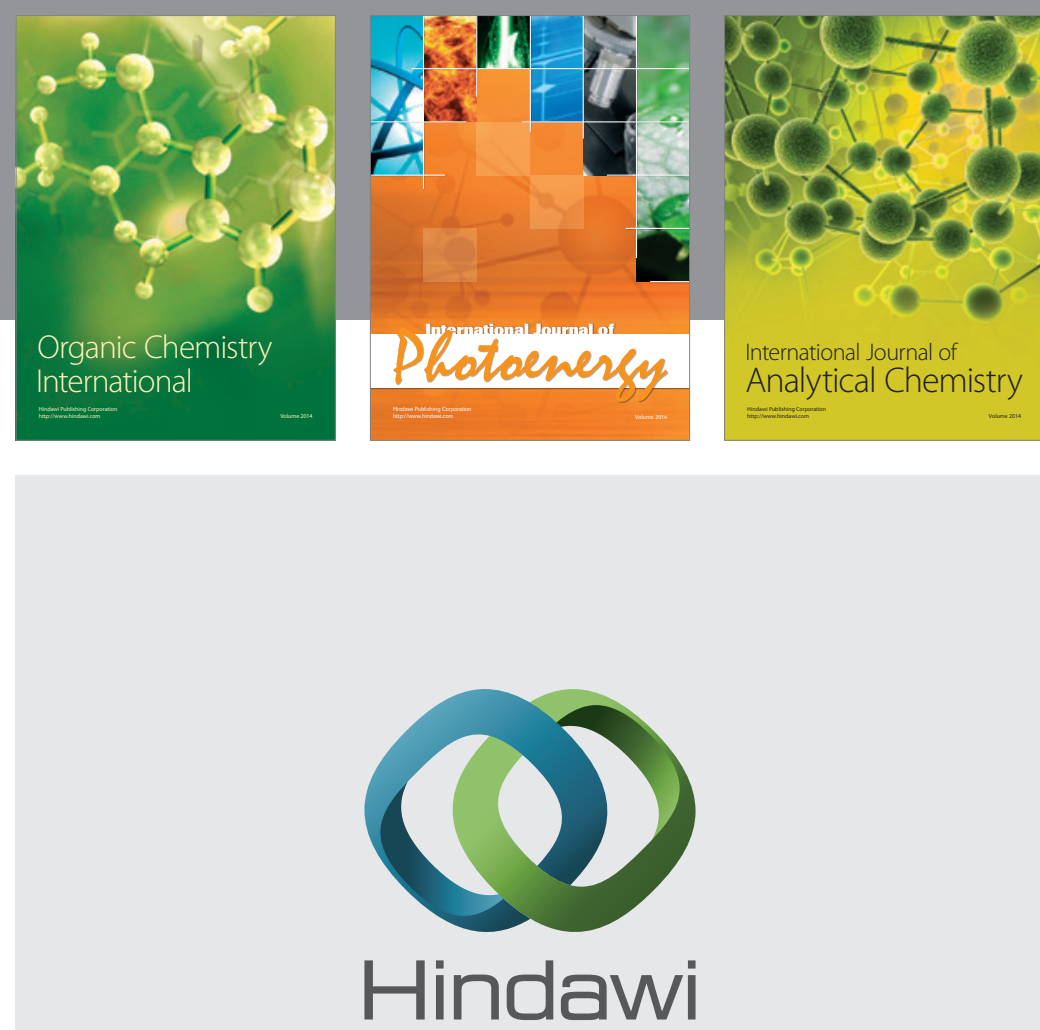

Submit your manuscripts at

http://www.hindawi.com
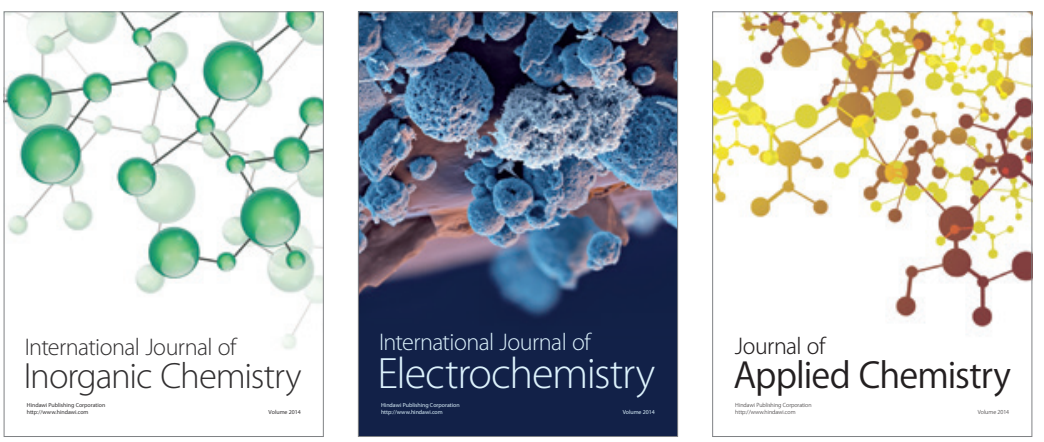

Journal of

Applied Chemistry
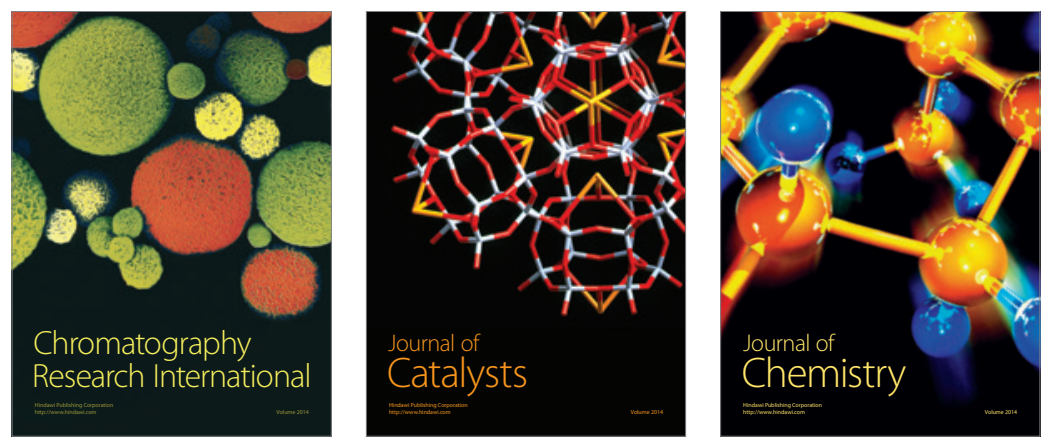
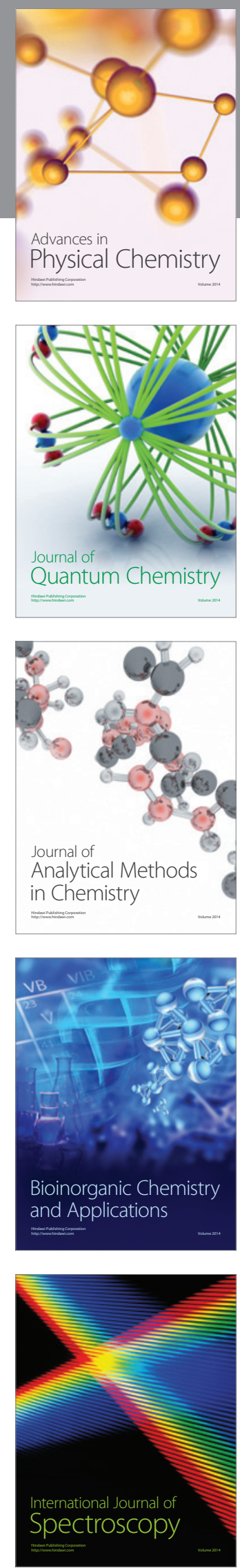